\title{
Type 2 diabetes and glycemic traits are not causal factors of osteoarthritis: A two-sample Mendelian randomization analysis
}

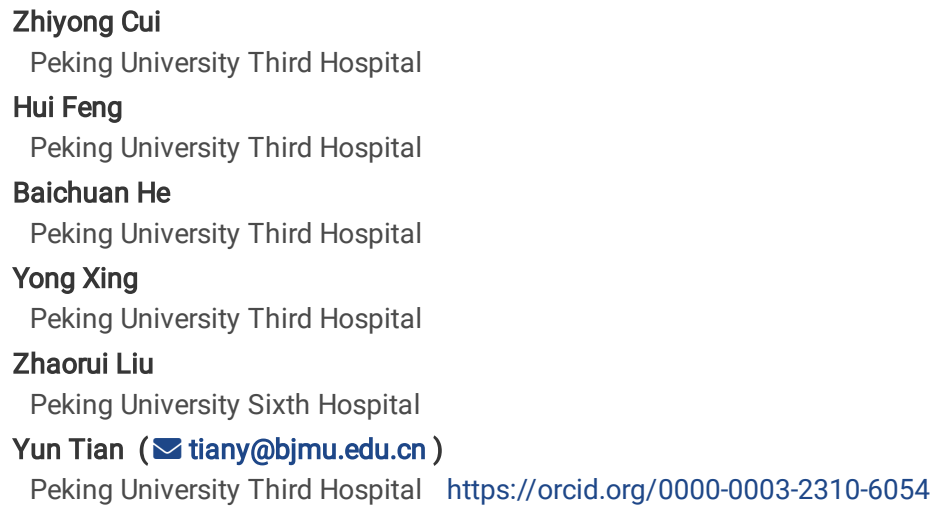

Research article

Keywords: osteoarthritis, type 2 diabetes, fasting glucose, 2-hour postprandial glucose, Mendelian randomization

Posted Date: June 23rd, 2020

DOI: https://doi.org/10.21203/rs.3.rs-37242/v1

License: @ (i) This work is licensed under a Creative Commons Attribution 4.0 International License. Read Full License

Version of Record: A version of this preprint was published at Frontiers in Genetics on January 13th, 2021. See the published version at https://doi.org/10.3389/fgene.2020.597876. 


\section{Abstract}

Background: It remains unclear whether an increased risk of type 2 diabetes (T2D) affects the risk of osteoarthritis (OA).

Methods: Here, we used two-sample Mendelian randomization (MR) to obtain non-confounded estimates of the effect of T2D and glycemic traits on hip and knee OA. We identified single nucleotide polymorphisms (SNPs) strongly associated with T2D, fasting glucose (FG) and 2-hour postprandial glucose (2hGlu) from genome-wide association studies (GWAS). We used MR inverse variance weighted (IVW), the MR-Egger method, the weighted median (WM) and Robust Adjusted Profile Score (MR.RAPS) to reveal the associations of T2D, FG and 2hGlu with hip and knee OA risk. Sensitivity analyses were also conducted to verify whether heterogeneity and pleiotropy can bias the MR results.

Results: We did not find statistically significant causal effects of genetically increased T2D risk, FG and 2hGlu on hip and knee OA (e.g., T2D and hip OA, MREgger $\mathrm{OR}=0.9536,95 \% \mathrm{Cl} 0.5585$ to $1.6283, p=0.8629$ ). It was confirmed that horizontal pleiotropy was unlikely to bias the causality (e.g., $\mathrm{T} 2 \mathrm{D}$ and hip $\mathrm{OA}$, MR-Egger, intercept $=-0.0032, p=0.8518$ ). No evidence of heterogeneity was found between the genetic variants (e.g., T2D and hip OA, MR-Egger $Q=40.5481$, $R=0.1368, p=0.2389)$.

Conclusions: Our MR study did not support causal effects of a genetically increased T2D risk, FG and 2hGlu on hip and knee OA risk.

\section{Introduction}

Osteoarthritis (OA) and type 2 diabetes (T2D) are two pandemic chronic diseases and have significant impact on quality of life, social expenditure, and life expectancy [1,2]. OA is the most common chronic joint disease and its main characteristics are the loss of chronic irreversible articular cartilage [1,2]. T2D is one category of diabetes, which is a chronic metabolic syndrome characterized by increased blood glucose levels as a consequence of insulin resistance [3]. $\mathrm{OA}$ and T2D share common risk factors, such as obesity, physical activity and genetic factors $[1,3,4]$, which could explain why there was increasing prevalence of $\mathrm{OA}$ in T2D. Since the exact mechanism involving T2D and OA was still debatable, it was controversial whether there was a causal relationship between $T 2 D$ and $O A$.

Observational studies to estimate the causal inference have numerous inherent limitations, such as remaining limited to known and properly measured confounders [5]. Therefore, we used Mendelian randomization (MR), an application of the method of instrumental variables (IVs) to the analysis of genetic data to assess the causal associations of T2D and related glycemic traits (fasting glucose (FG) and 2-hour postprandial glucose (2hGlu)) with hip and knee OA. The genetic variants in MR are available with the progress of genome-wide association studies (GWAS) and high-throughput genomic technologies. In this study, we used single-nucleotide polymorphisms (SNPs) strongly associated with T2D and glycemic traits as IVs. We performed a two-sample MR and used statistical methods to obtain quantitative estimates to investigate the effect of T2D, FG, and 2hGlu upon hip and knee OA.

\section{Materials And Methods}

In our study, we selected SNPs as IVs for all exposures (T2D, FG and 2hGlu) and outcomes (hip and knee OA) from the IEU GWAS database, a database of genetic associations from GWAS summary datasets (https://gwas.mrcieu.ac.uk/) [6]. When target SNPs were not available in the outcome study, we used proxy SNPs that were in high linkage disequilibrium (LD) $\left(r^{2}>0.8\right)$ with the SNPs of interest. We selected the reference sample formed by the European ancestral individuals from the 1000 genomes project to estimate the allele frequency and LD level (http://www.internationalgenome.org/) [7]. The palindromic SNPs with intermediate allele frequencies (palindromic SNPs referred to the SNPs with A/T or G/C alleles and "intermediate allele frequencies" referred to $0.01<$ allele frequency<0.30) were excluded from the above selected instrument SNPs. SNPs a minor allele frequency (MAF) of < 0.01 were also excluded. We also calculated the F statistics for the SNPs to measure the strength of the instruments. IVs with an F statistic less than 10 were excluded and were often labelled as "weak instruments" [8]. These rigorously selected SNPs were used as the final instrumental SNPs for the subsequent MR analysis.

SNPs associated with T2D were derived from a meta-analysis of GWAS in a very large sample of T2D (62,892 cases and 596,424 controls) of European ancestry [9]. SNPs associated with FG and 2hGlu were derived from genome-wide association meta-analyses of up to 133,010 individuals, with males and females, of European ancestry without diabetes, performed by The Meta-Analyses of Glucose and Insulin-related traits Consortium (MAGIC) [10]. The summary-level data for the impact of the exposures-associated SNPs on hip and knee OA were extracted from a GWAS of European descent performed by Arthritis Research UK Osteoarthritis Genetics (arcOGEN) Consortium [11]. The arcOGEN case samples were collected and genotyped in two stages. The stage one samples were those who had primary $\mathrm{OA}$ of the hip or knee with radiographic Kellgren-Lawrence (KL) grade $\geq 2$, or clinical evidence of disease to a level requiring total joint replacement. The stage two samples were those with primary OA that was severe enough for the individual to require joint replacement of the hip or of the knee, with an additional small number who were collected as part of a randomized, placebo controlled trial of vitamin $D$ replacement where the ascertainment criterion was radiographic disease of the knee, with a KL grade $\geq 2$. All the two stage samples were of European origin [11]. The detailed characteristics of GWAS associated with exposures (T2D, FG and 2hGlu) and outcomes (hip and knee OA) are shown in Table S1 in the Supplementary Material.

We applied the two-sample MR to assess the role of exposures (T2D, FG and 2hGlu) in the susceptibility of outcomes (hip and knee OA). We assessed the independent association of SNPs with T2D, FG and $2 \mathrm{hGlu}$, and selected SNPs that were strongly associated $(p<5 \mathrm{E}-08)$ and independent inheritance $\left(r^{2}<0.01\right)$ without any LD with the exposures. Then, we obtained the effect estimates for the selected SNPs on hip and knee OA from the arcOGEN Consortium in 2012. The causal associations between exposures (T2D, FG and 2hGlu) and outcomes (hip and knee OA) were estimated with inverse variance weighted (IVW), MREgger and weighted median (WM). The IVW method uses a meta-analysis approach to combine the Wald ratios of the causal effects of each SNP and can provide the most precise estimates. However, it can be influenced by invalid IVs and pleiotropic effects. The WM estimate provides a reliable effect estimate 
of the causal effect when at least $50 \%$ of the weight in the analysis comes from effective IVs. MR-Egger regression is based on the assumption that the pleiotropic associations are independent, performs a weighted linear regression of the outcome coefficients on the exposure coeffcients. MR-Egger estimates may be inaccurate and can be strongly influenced by outlying genetic variants [12]. We also performed a recently developed method called the Robust Adjusted Profile Score (MR.RAPS) to estimate the causal effects, which can lead to a considerably higher statistical power than the conventional MR analysis can, which only uses a small set of strong instruments [13]. MR.RAPS considers the measurement error in SNP-exposure effects and is unbiased when there are many weak instruments, and is robust to systematic and idiosyncratic pleiotropy [13].

We used the IVW, WM and maximum likelihood methods to evaluate the heterogeneity between SNPs. The heterogeneity was quantified by Cochran $Q$ statistics and $P$ statistics [12]. Moreover, the causal directions were tested by MR-Steiger method between the exposures and outcomes [14]. Pleiotropy refers to the phenomenon in which a single locus affects multiple phenotypes. Horizontal pleiotropy arises when a genetic variant associates with more than one phenotype on separate pathways, which can invalidate the results from MR analyses $[15,16]$. In order to explore and adjust for horizontal pleiotropy, we evaluated the pleiotropic effects of T2D and glycemic traits on weight-associated factors, including body mass index (BMI), weight and obesity, as these confounding effects might distort the effects of T2D and glycemic traits on OA. Summary statistics for BMI were extracted from studies performed by the Genetic Investigation of ANthropometric Traits (GIANT) consortium [17], weight (male and female) from GIANT Consortium [18], and obesity from GIANT Consortium [19]. The detailed characteristics of studies associated with confounding factors are shown in Table S1. We assessed the potential associations between SNPs that were extracted for the MR analysis and those confounding factors.

Associations of the SNPs with two outcomes were considered statistically significant at a Bonferroni-corrected $p<0.05 /(2 \times N)$, and associations of the SNPs with the four confounding factors were considered statistically significant at a Bonferroni-corrected $p<0.05 /(4 \times N)$, with $N$ representing the number of SNPs in each exposure trait. In addition to evaluating the associations with the risk factors, we also performed MR-Egger regression to explore and adjust for horizontal pleiotropy, which was a method that can provide evidence for confounders that would distort the MR results. The intercept represents the average pleiotropic effect across the genetic variants. The results of the MR analyses were considered statistically significant at a Bonferroni-corrected $p<0.025$ (e.g., 0.05/2 outcomes). All statistical tests were two-sided and performed using the "TwoSampleMR" package for R language, version 3.6 .1 (R Foundation for Statistical Computing, Vienna, Austria).

\section{Results}

\section{Causality Between T2D and OA}

For T2D, we used forty genomewide significant ( $p<5 \mathrm{E}-08$ ) SNPs associated with increased T2D risk identified in the largest meta-analysis of T2D GWAS studies [9]. For each of the susceptibility variants for T2D, we sought summary-level data for OA from the GWAS performed by arcOGEN Consortium. After removing three T2D variants (rs1333039, rs6494307, rs7619041) that were palindromic with intermediate allele frequencies, thirty-seven SNPs remained to perform the MR analysis for hip and knee OA. None of the thirty-seven SNPs were significantly associated with OA outcomes ( $p<6.76 \mathrm{E}-04)($ e.g., $0.05 / 74)$. Seven of the thirty-seven T2D variants (rs1061813, rs13239186, rs1801214, rs2493394, rs6066138, rs6515236 and rs7240767) was absent from the arcOGEN study. Therefore, we used seven proxy SNPs (rs1353258, rs10276758, rs1046314, rs5025718, rs6066149, rs6048114 and rs9948462) respectively that were in high LD $\left(r^{2}>0.8\right)$ with the seven SNPs of interest in our analysis (Table S2). For these IVs, all the F values were larger than 10 , ranging from 30.6746 to 256.3266 , with an average $F$ value of 51.9134 .

In our analysis using the full set of 37 SNPs, we did not find causal associations of per unit increase in the log-odds of having T2D with risk changes of having OA, based on IVW, WM, MR-Egger regression, and MR.RAPS methods at the Bonferroni-corrected significance threshold $p<0.025$ (e.g., $0.05 / 2$ ). (For hip $\mathrm{OA}$, MR-Egger OR=0.9536, $95 \% \mathrm{Cl} 0.5585$ to $1.6283, p=0.8629$; IVW OR $=0.9086,95 \% \mathrm{Cl} 0.7596$ to $1.0869, p=0.2945 ; \mathrm{WM}$ OR=0.9210,95\% $\mathrm{Cl} 0.7010$ to 1.2100, $p=0.5545$; MR.RAPS OR=0.8970, $95 \% \mathrm{Cl} 0.7337$ to $1.0967, p=0.2892$. For knee OA, MR-Egger OR=1.3162, $95 \% \mathrm{Cl} 0.8010$ to $2.1628, p=0.2856$; IVW $\mathrm{OR}=1.0085,95 \% \mathrm{Cl} 0.8521$ to $1.1921, p=0.9208$; WM OR=1.0000, $95 \% \mathrm{Cl} 0.7673$ to $1.3032, p=1.0000$; MR.RAPS OR=1.0076, $95 \% \mathrm{Cl} 0.8464$ to 1.1996 , $p=0.9319$.) (Table 1 and Figure 1). We assessed the horizontal pleiotropy by checking the association of T2D associated SNPs with confounders, and no significant association signal was detected among the thirty-seven SNPs we selected at the Bonferroni-corrected significance threshold $p<3.38 \mathrm{E}-04$ (e.g., $0.05 / 148$ ) (Table S3). We also assessed the horizontal pleiotropy with the MR-Egger regression and found no horizontal pleiotropy would bias the causality with hip OA (intercept $=-0.0032, p=0.8518$ ) and knee OA (intercept $=-0.0176, p=0.2720$ ) (Table 2). The heterogeneity test demonstrated that there is no evidence of heterogeneity in the MR analysis. (For hip OA, MR-Egger $Q=40.5481, P=0.1368, p=0.2389$; IVW $Q=40.5892, P=0.1131, p=0.2752$; Maximum likelihood $Q=40.5675, P=0.1126, p=0.2759$. For knee OA, MR-Egger $Q=30.9104, P_{<}<0.0001, p=0.6659$; IVW $Q=32.1561, P_{<}<.0001, p=0.6520$; Maximum likelihood $Q=32.1560, R<0.0001, p=0.6520$.) (Table 2). The MR-Steiger results supported the causal direction between T2D and OA traits (Table 1).

\section{Causality Between FG in non-diabetic individuals and OA}

Based on independent and LD analyses, we selected fifteen genomewide significant ( $p<5 \mathrm{E}-08$ ) SNPs associated with FG in non-diabetic individuals to analyze the causality with hip and knee OA, no palindromic SNPs were found. None of the fifteen SNPs were significantly associated with OA outcomes $(p<0.0017)$ (e.g., 0.05/30) (Table S4). Five target FG variants (rs2191349, rs3783347, rs479661, rs6072275 and rs6975024) were not available in the arcOGEN study. Therefore, we used five proxy SNPs (rs6947830, rs12882934, rs525428, rs2228246 and rs2908282) respectively that were in high LD ( $\left.r^{2}>0.8\right)$ with the five SNPs of interest in our analysis (Table S4). The F values of the fifteen SNPs ranged from 32.6531 to 806.5600 , with an average value of 150.5130 .

No evidence supported that the genetically-increased FG was causally associated with the hip and knee OA risk changes in non-diabetic individuals based on IVW, WM, MR-Egger regression and MR.RAPS methods ( $p \otimes 0.025$ ). (For hip OA, MR-Egger OR=0.5584, 95\% Cl 0.1741 to 1.7909, $p=0.3450$; IVW OR=0.5675, 95\% 
$\mathrm{Cl} 0.3143$ to $1.0248, p=0.0603$; WM OR=0.4772, 95\% $\mathrm{Cl} 0.2400$ to $0.9487, p=0.0348$; MR.RAPS OR=0.5712, 95\% $\mathrm{Cl} 0.3099$ to $1.0527, p=0.0726$. For knee OA, MR-Egger OR=0.4623, $95 \% \mathrm{Cl} 0.1447$ to $1.4771, p=0.2156$; IVW OR=0.7278, $95 \% \mathrm{Cl} 0.3947$ to $1.3418, p=0.3087$; WM OR=0.4896, $95 \% \mathrm{Cl} 0.2446$ to 0.9802 , $p=0.0438$; MR.RAPS OR=0.7247, $95 \% \mathrm{Cl} 0.3682$ to $1.4265, p=0.3514$.) (Table 1 and Figure 2 ). None of the fifteen SNPs were significantly associated with known confounders at the Bonferroni-corrected significance threshold ( $p<8.33 \mathrm{E}-04)$ (e.g., 0.05/60) (Table S5). We also conducted the MR-Egger regression to assess the horizontal pleiotropy, and the results revealed that the horizontal pleiotropy was unlikely to bias the causality with hip $0 \mathrm{~A}$ (intercept=0.0006, $p=0.9748$ ) and knee OA (intercept $=0.0170, p=0.3830$ ) (Table 2).

We also found no significant heterogeneity between FG and OA. (For hip OA, MR-Egger $Q=17.7654, P=0.2682, p=0.1666$; IVW $Q=17.7668, P=0.2120, p=0.2176$; Maximum likelihood $Q=17.7374, R=0.2107, p=0.2190$. For knee OA, MR-Egger $Q=19.7632, R=0.3437, p=0.1013$; IVW $Q=21.0027, R=0.3334, p=0.1016$; Maximum likelihood $Q=20.9936, P=0.3331, p=0.1018$.) (Table 2). The MR-Steiger directionality test showed that the direction of the IV estimates between higher level of FG and OA risk in non-diabetic individuals remained largely unchanged (Table 1).

\section{Causality Between $2 \mathrm{hGlu}$ in non-diabetic individuals and $\mathrm{OA}$}

We chose three independent SNPs associated with $2 \mathrm{hGlu}$ in European ancestry from summary statistics data sets of GWAS meta-analyses, and no palindromic SNPs were found. None of the three SNPs were significantly associated with OA outcomes $(p<0.0083)($ e.g., $0.05 / 6)$. Two target SNPs (rs1019503 and rs6975024) were unavailable in the outcome database. Therefore, two proxy SNPs (rs1981846 and rs2908282) were chosen in the 1000 Genomes Project-imputed genotype data that were in high LD $\left(r^{2}>0.8\right)$ with the two target SNPs respectively (Table S6). The F values of the three SNPs were $32.8017,33.5180$ and 39.0625 , with an average value of 35.1274 .

Our results did not suggest causal associations of genetically-increased $2 \mathrm{hGlu}$ with hip and knee OA risk changes in non-diabetic individuals ( $p \varangle 0.025$ ). (For hip OA, MR-Egger OR=0.2509, $95 \% \mathrm{Cl} 0.0338$ to $1.8614, p=0.4054$; IVW OR=0.8368, $95 \% \mathrm{Cl} 0.5154$ to $1.3586, p=0.2473$; WM OR=0.7844, $95 \% \mathrm{Cl} 0.4320$ to 1.4242, $p=0.4249$; MR.RAPS OR=0.8311, $95 \% \mathrm{Cl} 0.4939$ to $1.3985, p=0.4860$. For knee OA, MR-Egger OR=0.4928, $95 \% \mathrm{Cl} 0.0759$ to $3.1997, p=0.5938 ; \mathrm{IVW}$ $\mathrm{OR}=0.8362,95 \% \mathrm{Cl} 0.5292$ to $1.3212, p=0.4433$; WM OR=0.8345, $95 \% \mathrm{Cl} 0.4847$ to $1.4368, p=0.5140$; MR.RAPS OR=0.8356, $95 \% \mathrm{Cl} 0.5210$ to 1.3402 , $p=0.4562$.) (Table 1 and Figure 3). We conducted the MR-Egger regression to assess the pleiotropy, and the results revealed that the horizontal pleiotropy was unlikely to bias the causality with hip OA (intercept $=0.1051, p=0.4386$ ) and knee OA (intercept $=0.0451, p=0.6695)($ Table 1 ).

The associations between these genetic variants and confounding factors were analyzed. None of the three genetic variants were significantly associated with the confounding factors mentioned above at the Bonferroni-corrected significance threshold $(p<0.0042)($ e.g., $0.05 / 12)($ Table S7). Cochran's $Q$ value and the $\mathcal{R}$ value indicated no evidence of heterogeneity between IV estimates with IVW, MR-Egger and maximum likelihood methods (For hip OA, MR-Egger $Q=0.5059, R \otimes 0.0001, p=0.4769$; IVW $Q=1.9800, R \otimes 0.0001, p=0.3716$; Maximum likelihood $Q=1.9699, R \otimes 0.0001, p=0.3735$. For knee $0 \mathrm{~A}, \mathrm{MR}-\mathrm{Egger} Q=0.0485$, $R<0.0001, p=0.8257$; IVW $Q=0.3749, R<0.0001, p=0.8291$; Maximum likelihood $Q=0.3730, R<0.0001, p=0.8299$.) (Table 2). The MR-Steiger results supported the causal direction between the exposures and outcomes (Table 1).

\section{Discussion}

To our knowledge, this is the first MR study on the effect of T2D and other glycemic traits on OA. We did not distinguish statistical causality between exposures and outcomes based on our MR results. The F values of IVs indicated that the variables satisfy the strong relevance assumption of MR and that the instrument bias was weak and could not substantially influence the estimations of causal effects. We used the MR-Egger method to detect and adjust for pleiotropy of the genetic variants. We also performed heterogeneity and did not find significant heterogeneity between SNPs, which indicated the reliability of the MR results. Some studies reported the similar results that there was no evidence to support the causal associations between T2D and OA. Frey N et al [20] conducted one case-control study and provided evidence that T2D is not an independent risk factor for hand OA regardless of T2D severity, duration, or pharmacological treatment. Funck-Brentano T et al [21] analyzed the individual-level data in UK Biobank study and performed MR analysis. They found no significant causality for T2D with all OA, knee OA, hip OA and hand OA. Zengini E et al [22] also found no causal association of T2D with self-reported OA or hospital-diagnosed OA with the MR analysis. On the contrary, many studies reported the suggestive evidence to support the associations between T2D and $\mathrm{OA}$. Eymard F et al [23] demonstrated that T2D was a predictor of joint space reduction in men with established knee OA. Davies-Tuck ML et al [24] reported the evidence that increased FG concentration in a non-diabetic individuals was associated with adverse structural changes at the knee in women based on one prospective cohort study. William MF et al [25] executed one meta-analysis including ten observational studies with 16,742 patients in total. They revealed that T2D was associated with radiographic and symptomatic OA even after controlling the BMI and weight. Schett $\mathrm{G}$ et al [26] reported that T2D could predict the progress of OA, independent of age and BMI, based on one cohort study followed over 20 years.

Some studies provided a few suggestive pathophysiological mechanisms for the development of OA in T2D patients. One of them was hyperglycemiainduced accelerated synthesis of Advanced Glycation End products (AGEs), which leads to an increase in oxidative stress. These AGEs have been regarded as one of the factors responsible for healing impairment and loss of elasticity of cartilage [27]. Another mechanism was that chronic high glucose environment had noxious effects on chondrocytes metabolism [28]. High glucose environment would induce diabetic cartilages to produce more interleukin-6 and prostaglandin E2. High glucose exposure also increased the metalloproteinases production especially in human OA chondrocytes and decreased the production of collagen II. Vaamonde-Garcia C et al [29] demonstrated that high glucose environment favoured the suppession of haem oxygenase-1, which led to an increase in the oxidative stress and cartilage damage. Chen $\mathrm{YJ}$ et al [30] found that high glucose diminished the synthesis of type II collagen and PPARy by chondrocytes, which could result in the development of cartilage defects.

Although many studies suggested the associations, those evidences were too weak to indicate the causal associations between T2D and OA. Confounders might interfere with the associations between T2D and OA. Since T2D and OA share common risk factors, some studies even suggested that OA was the

Page $4 / 10$ 
component of metabolic syndrome [26], metabolic factors such as obesity and inflammatory factors, physical activity and diabetic medication might have an impact on the OA. For example, obesity is a pandemic condition defined as the abnormal or the excessive accumulation of fat, which is characteristic of T2D [31]. Obesity would promote the progress of OA through mechanical load and inflammatory reaction. Mechanical load means that the increase load of weight-bearing joint caused by obesity could accelerate the development of OA. Inflammatory reaction indicated that the increased systemic and local inflammation caused by obesity would damage the integrity of the extracellular matrix of cartilage [1,4]. Furthermore, some confounders that cannot be entirely ruled out such as socioeconomic status, occupation and nutrition also had impact on the association between T2D and OA [20]. Some studies [26] used joint arthroplasty due to $\mathrm{OA}$ as the study endpoint, which precludes the ability to assess temporality, because the joint arthroplasty was the terminal event of OA. This would be the reason that affected the association between T2D and OA. Besides》reverse causation bias between T2D and OA [32] would also limit the ability to provide causal estimates of the effect of exposures on outcomes in the observational studies.

The present study has several limitations. The criteria for OA were limited in the GWAS included in the study. The radiographic OA or the mild symptomatic OA were not included. Additionally, there were only two types of OA, hip and knee OA involved in the study. The hand OA was not analyzed in the MR study, which might distinguish in pathogenesis mechanism from hip and knee OA due to the absence of weight-bearing factors. Our analysis included only three SNPs as IVs on $2 \mathrm{hGlu}$ and might had limited power to detect causal associations between $2 \mathrm{hGlu}$ and OA. The sample included in the exposures and outcomes were of European ancestry, which could mitigate the population stratification. However, the conclusions based on the European sample were not representative of other ancestries, like Asians and Americans. Moreover, we only evaluated the associations between SNPs and weight-associated confounders due to the limited publicly available GWAS databases. The associations between these instruments and other potential confounders such as physical activity, were not evaluated in our study.

\section{Conclusion}

In summary, our two-sample MR analysis did not suggest the significant causal effects of genetic increases in type 2 diabetes risk, fasting glucose and 2hour postprandial glucose with hip and knee osteoarthritis. The complicated effects of type 2 diabetes risk, fasting glucose and 2-hour postprandial glucose with osteoarthritis might be influenced by other confounding factors, which still need further investigation in the future. In addition, future studies should additionally seek to investigate the effect of type 2 diabetes and glycemic traits on hand osteoarthritis.

\section{Abbreviations}

osteoarthritis (OA); type 2 diabetes (T2D); Mendelian randomization (MR); instrumental variables (IVs); fasting glucose (FG); 2-hour postprandial glucose (2hGlu); genome-wide association studies (GWAS); single-nucleotide polymorphisms (SNPs); linkage disequilibrium (LD); minor allele frequency (MAF); The Meta-Analyses of Glucose and Insulin-related traits Consortium (MAGIC); Arthritis Research UK Osteoarthritis Genetics (arcOGEN); Kellgren-Lawrence (KL); inverse variance weighted (IVW); weighted median (WM); MR Robust Adjusted Profile Score (MR.RAPS); body mass index (BMI); Genetic Investigation of ANthropometric Traits (GIANT)

\section{Declarations}

\section{Ethics approval and consent to participate}

Not applicable

\section{Consent for publication}

Not applicable

\section{Contributions}

Yun Tian, Zhiyong Cui and Baichuan He conceptualized and designed the study. Zhiyong Cui and Hui Feng provided the "TwoSampleMR" package codes in R language and analyzed the data in the study. Zhiyong Cui drafted the manuscript. Yong Xing and Zhaorui Liu gave constructive suggestions when writing the manuscript. All the authors have read the manuscript.

\section{Funding}

This work was supported by grants from Beijing Municipal Science and Technology Commission (Z181100001718195).

\section{Competing interests}

The authors declare that they have no conflict of interest.

\section{Availability of data and materials}

Summary-level data for genetic associations with T2D, FG and 2hGlu, hip and knee OA, BMI, weight and obesity were from the T2D dataset (DIAbetes Genetics Replication And Meta-analysis (DIAGRAM), Genetic Epidemiology Research on Aging (GERA) and UK Biobank (UKB)), The Meta-Analyses of Glucose and Insulin-related traits Consortium (MAGIC), Arthritis Research UK Osteoarthritis Genetics (arcOGEN) Consortium and the Genetic Investigation of ANthropometric Traits (GIANT) consortium. 


\section{Acknowledgments}

We thank all the participants and researchers of the studies included in the current analyses.

\section{References}

1. Martel-Pelletier J, Barr AJ, Cicuttini F et al. Nat Rev Dis Primers. 2016;2:16072. http://10.1038/nrdp.2016.72

2. Hunter D, Bierma-Zeinstra S. Osteoarthritis. Lancet. 2019;393(10182):1745-1759. http://10.1016/S0140-6736(19)30417-9

3. Chatterjee S, Khunti K, Davies MJ. Type 2 diabetes. Lancet. 2017;389(10085):2239-2251. http://10.1016/S0140-6736(17)30058-2

4. Reyes C, Leyland K, Peat G et al. Association between overweight and obesity and risk of clinically diagnosed knee, hip, and hand osteoarthritis: a population-based cohort study. Arthritis Rheumatol. 2016;68(8):1869-1875. http://10.1002/art.39707

5. Greenland S, Morgenstern H. Confounding in health research. Annu Rev Publ Health. 2001;22:189-212. http://10.1146/annurev.publhealth.22.1.189

6. Hemani G, Zheng J, Elsworth B et al. The MR-Base platform supports systematic causal inference across the human phenome. Elife. $2018 ; 7$ : e34408. http://10.7554/eLife.34408

7. 1000 Genomes Project Consortium, Abecasis GR, Altshuler D et al. A map of human genome variation from population-scale sequencing. Nature. 2010;467(7319):1061-1073. http://10.1038/nature09534

8. Burgess S, Small DS, Thompson SG. A review of instrumental variable estimators for Mendelian randomization. Stat Methods Med Res. 2015;26(5):2333-2355. http://10.1177/0962280215597579

9. Xue A, Wu Y, Zhu Z, Zhang F et al. Genome-wide association analyses identify 143 risk variants and putative regulatory mechanisms for type 2 diabetes. Nat Commun. 2018;9(1):2941. http://10.1038/s41467-018-04951-w

10. Scott RA, Lagou V, Welch RP et al. Large-scale association analyses identify new loci influencing glycemic traits and provide insight into the underlying biological pathways. Nat Genet. 2012;44(9):991-1005. http://10.1038/ng.2385

11. Zeggini E, Panoutsopoulou K, Southam L et al. Identification of new susceptibility loci for osteoarthritis (arcOGEN): a genome-wide association study. Lancet. 2012;380(9844):815-823. http://10.1016/S0140-6736(12)60681-3

12. Bowden J, Del Greco M F, Minelli C et al. Assessing the suitability of summary data for two-sample Mendelian randomization analyses using MR-Egger regression: the role of the I2 statistic. Int J Epidemiol. 2016;45(6):1961-1974. http://10.1093/ije/dyw220

13. Zhao Q, Chen Y, Wang J et al. Powerful three-sample genome-wide design and robust statistical inference in summary-data Mendelian randomization. Int J Epidemiol. 2019;48(5):1478-1492. http://10.1093/ije/dyz142

14. Hemani G, Tilling K, Davey Smith G. Orienting the causal relationship between imprecisely measured traits using GWAS summary data. PLOS Genetics. 2017;13(11):e1007081. http://10.1371/journal.pgen.1007081

15. Stearns FW. One hundred years of pleiotropy: a retrospective. Genetics. 2010;186(3):767-773. http://10.1534/genetics.110.122549

16. Larsson S, Michaëlsson K, Burgess S. Mendelian randomization in the bone field. Bone. 2019;126:51-58. http://10.1016/j.bone.2018.10.011

17. Locke AE, Kahali B, Berndt SI et al. Genetic studies of body mass index yield new insights for obesity biology. Nature. 2015;518(7538):197-206. http://10.1038/nature14177

18. Randall JC, Winkler TW, Kutalik Z et al. Sex-stratified genome-wide association studies including 270,000 individuals show sexual dimorphism in genetic loci for anthropometric traits. Plos Genet. 2013;9(6):e1003500. http://10.1371/journal.pgen.1003500

19. Berndt SI, Gustafsson S, Mägi R et al. Genome-wide meta-analysis identifies 11 new loci for anthropometric traits and provides insights into genetic architecture. Nat Genet. 2013;45(5):501-512. http://10.1038/ng.2606

20. Frey N, Hügle T, Jick SS et al. Type II diabetes mellitus and incident osteoarthritis of the hand: a population-based case-control analysis. Osteoarthritis Cartilage.2016;24(9):1535-1540. http://10.1016/j.joca.2016.04.005.

21. Funck-Brentano T, Nethander M, Movérare-Skrtic S et al. Causal factors for knee, hip, and hand osteoarthritis: A Mendelian randomization study in the UK Biobank. Arthritis Rheumatol. 2019;71(10):1634-1641. http://10.1002/art.40928

22. Zengini E, Hatzikotoulas K, Tachmazidou I et al. Genome-wide analyses using UK Biobank data provide insights into the genetic architecture of osteoarthritis. Nat Genet. 2018;50(4):549-558. http://10.1038/s41588-018-0079-y

23. Eymard F, Parsons C, Edwards MH et al. Diabetes is a risk factor for knee osteoarthritis progression. Osteoarthritis Cartilage. 2015;23(6):851-859. http://10.1016/j.joca.2015.01.013

24. Davies-Tuck ML, Wang Y, Wluka AE et al. Increased fasting serum glucose concentration is associated with adverse knee structural changes in adults with no knee symptoms and diabetes. Maturitas. 2012;72(4):373-378. http://10.1016/j.maturitas.2012.05.013

25. Williams MF, London DA, Husni EM et al. Type 2 diabetes and osteoarthritis: a systematic review and meta-analysis. J Diabetes Complications. 2016;30(5):944-950. http://10.1016/j.jdiacomp.2016.02.016

26. Schett G, Kleyer A, Perricone C et al. Diabetes is an independent predictor for severe osteoarthritis: Results from a longitudinal cohort study. Diabetes Care. 2013;36(2):403-409. http://10.2337/dc12-0924

27. DeGroot J, Verzijl N, Jacobs KM et al. Accumulation of advanced glycation endproducts reduces chondrocyte-mediated extracellular matrix turnover in human articular cartilage. Osteoarthritis Cartilage. 2001;9(8):720-726. http://10.1053/joca.2001.0469

28. Laiguillon MC, Courties A, Houard X et al. Characterization of diabetic osteoarthritic cartilage and role of high glucose environment on chondrocyte activation: toward pathophysiological delineation of diabetes mellitus-related osteoarthritis. Osteoarthritis Cartilage. 2015;23(9):1513-1522.

Page 6/10 
http://10.1016/j.joca.2015.04.026

29. Vaamonde-Garcia C, Courties A, Pigenet A et al. The nuclear factor-erythroid 2-related factor/heme oxygenase-1 axis is critical for the inflammatory features of type 2 diabetes-associated osteoarthritis. J Biol Chem. 2017;292(35):14505-14515. http://10.1074/jbc.M117.802157

30. Chen Y, Chan DC, Lan KC et al. PPARyis involved in the hyperglycemia-induced inflammatory responses and collagen degradation in human chondrocytes and diabetic mouse cartilages. J Orthop Res 2015;33(3):373-381. http://10.1002/jor.22770

31. Kolb H, Martin S. Environmental/lifestyle factors in the pathogenesis and prevention of type 2 diabetes. BMC Med. 2017;15(1):131. http://10.1186/s12916-017-0901-x

32. Kendzerska T, King LK, Lipscombe L et al.The impact of hip and knee osteoarthritis on the subsequent risk of incident diabetes: a population-based cohort study. Diabetologia. 2018;61(11):2290-2299. http://10.1007/s00125-018-4703-2

\section{Tables}

Table 1. MR estimates from each method of assessing the causal effects of T2D, FG and 2hGlu on OA risk.

\begin{tabular}{|c|c|c|c|c|c|c|c|c|c|c|c|}
\hline \multirow{2}{*}{$\begin{array}{l}\text { Exposure } \\
\text { traits }\end{array}$} & \multirow{2}{*}{$\begin{array}{l}\text { MR } \\
\text { methods }\end{array}$} & \multicolumn{5}{|l|}{ Hip OA } & \multicolumn{5}{|l|}{ Knee OA } \\
\hline & & $\begin{array}{l}\text { Number } \\
\text { of } \\
\text { SNPs }\end{array}$ & OR $(95 \% \mathrm{Cl})$ & SE & $\begin{array}{l}\text { MR } p \text { - } \\
\text { value }\end{array}$ & $\begin{array}{l}\text { MR- } \\
\text { Steiger } \\
\text { test }\end{array}$ & $\begin{array}{l}\text { Number } \\
\text { of } \\
\text { SNPs }\end{array}$ & OR (95\% Cl) & SE & $\begin{array}{l}\text { MR } p \text { - } \\
\text { value }\end{array}$ & $\begin{array}{l}\text { MR- } \\
\text { Steiger } \\
\text { test }\end{array}$ \\
\hline \multirow[t]{4}{*}{ T2D } & $\begin{array}{l}\text { MR- } \\
\text { Egger }\end{array}$ & 37 & $\begin{array}{l}0.9536 \\
(0.5585 \sim 1.6283)\end{array}$ & 0.2730 & 0.8629 & \multirow{4}{*}{ 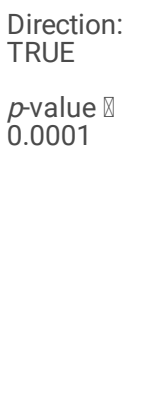 } & 37 & $\begin{array}{l}1.3162 \\
(0.8010 \sim 2.1628)\end{array}$ & 0.2534 & 0.2856 & \multirow{4}{*}{$\begin{array}{l}\text { Direction: } \\
\text { TRUE } \\
p \text {-value } \rrbracket \\
0.0001\end{array}$} \\
\hline & $\begin{array}{l}\text { Inverse } \\
\text { variance } \\
\text { weighted }\end{array}$ & 37 & $\begin{array}{l}0.9086 \\
(0.7596 \sim 1.0869)\end{array}$ & 0.0914 & 0.2945 & & 37 & $\begin{array}{l}1.0085 \\
(0.8521 \sim 1.1921)\end{array}$ & 0.0853 & 0.9208 & \\
\hline & $\begin{array}{l}\text { Weighted } \\
\text { median }\end{array}$ & 37 & $\begin{array}{l}0.9210 \\
(0.7010 \sim 1.2100)\end{array}$ & 0.1393 & 0.5545 & & 37 & $\begin{array}{l}1.0000 \\
(0.7673 \sim 1.3032)\end{array}$ & 0.1351 & 1.0000 & \\
\hline & $\begin{array}{l}\text { Robust } \\
\text { adjusted } \\
\text { profile } \\
\text { score }\end{array}$ & 37 & $\begin{array}{l}0.8970 \\
(0.7337 \sim 1.0967)\end{array}$ & 0.1025 & 0.2892 & & 37 & $\begin{array}{l}1.0076 \\
(0.8464 \sim 1.1996)\end{array}$ & 0.0890 & 0.9319 & \\
\hline \multirow[t]{4}{*}{ FG } & $\begin{array}{l}\text { MR- } \\
\text { Egger }\end{array}$ & 15 & $\begin{array}{l}0.5584 \\
(0.1741 \sim 1.7909)\end{array}$ & 0.5946 & 0.3450 & \multirow{4}{*}{$\begin{array}{l}\text { Direction: } \\
\text { TRUE } \\
p \text {-value } \rrbracket \\
0.0001\end{array}$} & 15 & $\begin{array}{l}0.4623 \\
(0.1447 \sim 1.4771)\end{array}$ & 0.5926 & 0.2156 & \multirow{4}{*}{$\begin{array}{l}\text { Direction: } \\
\text { TRUE } \\
p \text {-value } \rrbracket \\
0.0001\end{array}$} \\
\hline & $\begin{array}{l}\text { Inverse } \\
\text { variance } \\
\text { weighted }\end{array}$ & 15 & $\begin{array}{l}0.5675 \\
(0.3143 \sim 1.0248)\end{array}$ & 0.3015 & 0.0603 & & 15 & $\begin{array}{l}0.7278 \\
(0.3947 \sim 1.3418)\end{array}$ & 0.3122 & 0.3087 & \\
\hline & $\begin{array}{l}\text { Weighted } \\
\text { median }\end{array}$ & 15 & $\begin{array}{l}0.4772 \\
(0.2400 \sim 0.9487)\end{array}$ & 0.3506 & 0.0348 & & 15 & $\begin{array}{l}0.4896 \\
(0.2446 \sim 0.9802)\end{array}$ & 0.3514 & 0.0438 & \\
\hline & $\begin{array}{l}\text { Robust } \\
\text { adjusted } \\
\text { profile } \\
\text { score }\end{array}$ & 15 & $\begin{array}{l}0.5712 \\
(0.3099 \sim 1.0527)\end{array}$ & 0.3119 & 0.0726 & & 15 & $\begin{array}{l}0.7247 \\
(0.3682 \sim 1.4265)\end{array}$ & 0.3455 & 0.3514 & \\
\hline \multirow[t]{4}{*}{ 2hGlu } & $\begin{array}{l}\text { MR- } \\
\text { Egger }\end{array}$ & 3 & $\begin{array}{l}0.2509 \\
(0.0338 \sim 1.8614)\end{array}$ & 1.0225 & 0.4054 & \multirow{4}{*}{ 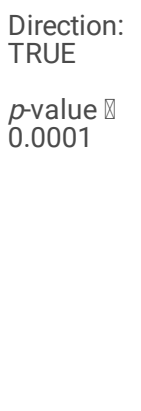 } & 3 & $\begin{array}{l}0.4928 \\
(0.0759 \sim 3.1997)\end{array}$ & 0.9544 & 0.5938 & \multirow{4}{*}{$\begin{array}{l}\text { Direction: } \\
\text { TRUE } \\
p \text {-value } \rrbracket \\
0.0001\end{array}$} \\
\hline & $\begin{array}{l}\text { Inverse } \\
\text { variance } \\
\text { weighted }\end{array}$ & 3 & $\begin{array}{l}0.8368 \\
(0.5154 \sim 1.3586)\end{array}$ & 0.2473 & 0.4712 & & 3 & $\begin{array}{l}0.8362 \\
(0.5292 \sim 1.3212)\end{array}$ & 0.2334 & 0.4433 & \\
\hline & $\begin{array}{l}\text { Weighted } \\
\text { median }\end{array}$ & 3 & $\begin{array}{l}0.7844 \\
(0.4320 \sim 1.4242)\end{array}$ & 0.3043 & 0.4249 & & 3 & $\begin{array}{l}0.8345 \\
(0.4847 \sim 1.4368)\end{array}$ & 0.2772 & 0.5140 & \\
\hline & $\begin{array}{l}\text { Robust } \\
\text { adjusted } \\
\text { profile } \\
\text { score }\end{array}$ & 3 & $\begin{array}{l}0.8311 \\
(0.4939 \sim 1.3985)\end{array}$ & 0.2655 & 0.4860 & & 3 & $\begin{array}{l}0.8356 \\
(0.5210 \sim 1.3402)\end{array}$ & 0.2410 & 0.4562 & \\
\hline
\end{tabular}

Table 2. Heterogeneity and pleiotropy analysis of T2D, FG and 2hGlu with hip and knee OA risk using different analytic methods. 


\begin{tabular}{|c|c|c|c|c|c|c|c|c|c|c|c|}
\hline \multirow{3}{*}{$\begin{array}{l}\text { Exposure } \\
\text { traits }\end{array}$} & \multirow{3}{*}{$\begin{array}{l}\text { MR } \\
\text { methods }\end{array}$} & \multicolumn{5}{|l|}{ Hip OA } & \multicolumn{5}{|l|}{ Knee OA } \\
\hline & & \multirow{2}{*}{$\begin{array}{l}\text { Cochran } \\
\text { Q } \\
\text { statistic }\end{array}$} & \multirow[t]{2}{*}{$R$} & \multirow{2}{*}{$\begin{array}{l}\text { Heterogeneity } \\
p \text {-value }\end{array}$} & \multicolumn{2}{|l|}{ MR-Egger } & \multirow{2}{*}{$\begin{array}{l}\text { Cochran } \\
\text { Q } \\
\text { statistic }\end{array}$} & \multirow[t]{2}{*}{ R } & \multirow{2}{*}{$\begin{array}{l}\text { Heterogeneity } \\
p \text {-value }\end{array}$} & \multicolumn{2}{|l|}{ MR-Egger } \\
\hline & & & & & Intercept & $\begin{array}{l}\text { Intercept } \\
p \text {-value }\end{array}$ & & & & Intercept & $\begin{array}{l}\text { Intercep } \\
p \text {-value }\end{array}$ \\
\hline \multirow[t]{3}{*}{$\mathrm{T} 2 \mathrm{D}$} & MR-Egger & 40.5481 & 0.1368 & 0.2389 & -0.0032 & 0.8518 & 30.9104 & $<0.0001$ & 0.6659 & -0.0176 & 0.2720 \\
\hline & $\begin{array}{l}\text { Inverse } \\
\text { variance } \\
\text { weighted }\end{array}$ & 40.5892 & 0.1131 & 0.2752 & & & 32.1561 & $<0.0001$ & 0.6520 & & \\
\hline & $\begin{array}{l}\text { Maximum } \\
\text { likelihood }\end{array}$ & 40.5675 & 0.1126 & 0.2759 & & & 32.1560 & $<0.0001$ & 0.6520 & & \\
\hline \multirow[t]{3}{*}{ FG } & MR-Egger & 17.7654 & 0.2682 & 0.1666 & 0.0006 & 0.9748 & 19.7632 & 0.3437 & 0.1013 & 0.0170 & 0.3830 \\
\hline & $\begin{array}{l}\text { Inverse } \\
\text { variance } \\
\text { weighted }\end{array}$ & 17.7668 & 0.2120 & 0.2176 & & & 21.0027 & 0.3334 & 0.1016 & & \\
\hline & $\begin{array}{l}\text { Maximum } \\
\text { likelihood }\end{array}$ & 17.7374 & 0.2107 & 0.2190 & & & 20.9936 & 0.3331 & 0.1018 & & \\
\hline \multirow[t]{3}{*}{ 2hGlu } & MR-Egger & 0.5059 & $<0.0001$ & 0.4769 & 0.1051 & 0.4386 & 0.0485 & $<0.0001$ & 0.8257 & 0.0451 & 0.6695 \\
\hline & $\begin{array}{l}\text { Inverse } \\
\text { variance } \\
\text { weighted }\end{array}$ & 1.9800 & $<0.0001$ & 0.3716 & & & 0.3749 & $<0.0001$ & 0.8291 & & \\
\hline & $\begin{array}{l}\text { Maximum } \\
\text { likelihood }\end{array}$ & 1.9699 & $<0.0001$ & 0.3735 & & & 0.3730 & $<0.0001$ & 0.8299 & & \\
\hline
\end{tabular}

\section{Figures}

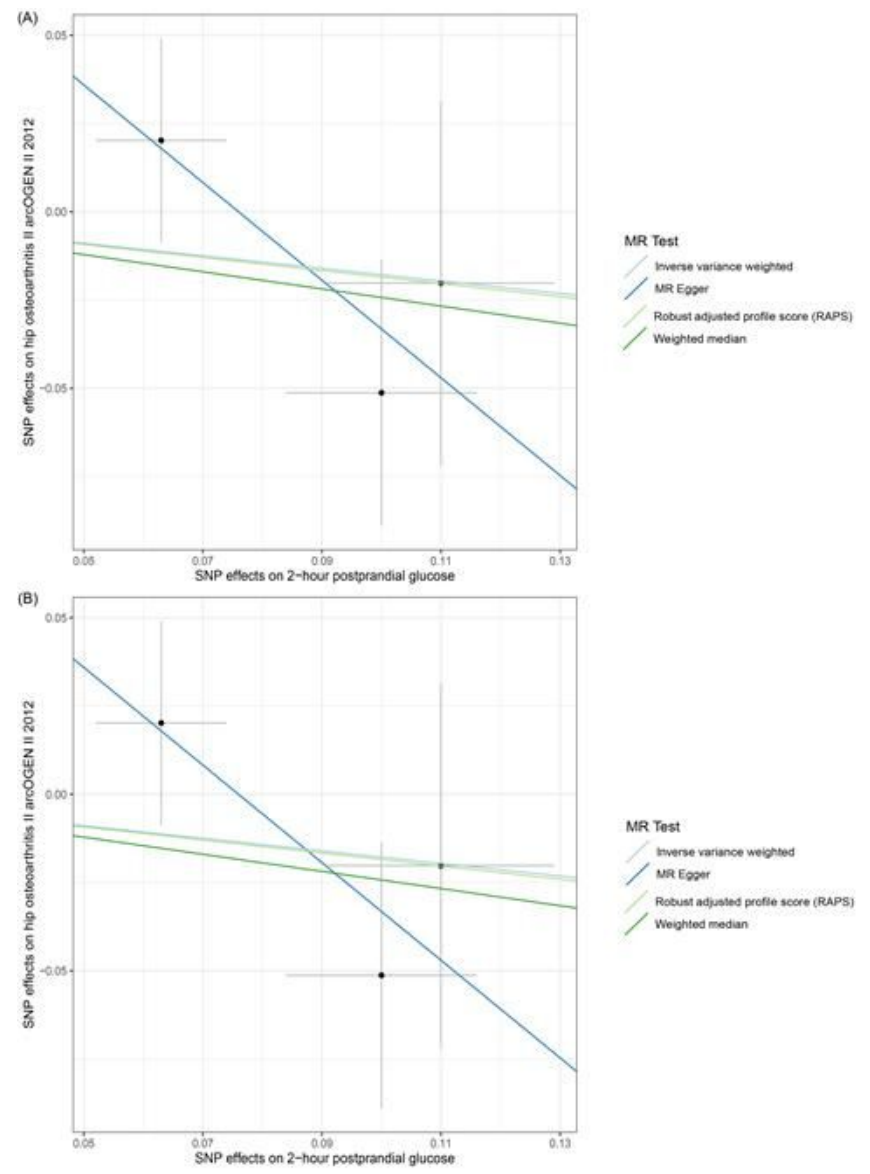

Figure 1 
Scatter plots of genetic associations with 2-hour postprandial glucose against osteoarthritis using different MR methods. A) 2-hour postprandial glucose and hip osteoarthritis results; B) 2-hour postprandial glucose and knee osteoarthritis results. The slopes of each line represent the causal association for each method. The light blue line represents the inverse variance weighted estimate, the light green line represents the MR.RAPS estimate, the dark blue line represents the MR-Egger estimate, and the dark green line represents the weighted median estimate.
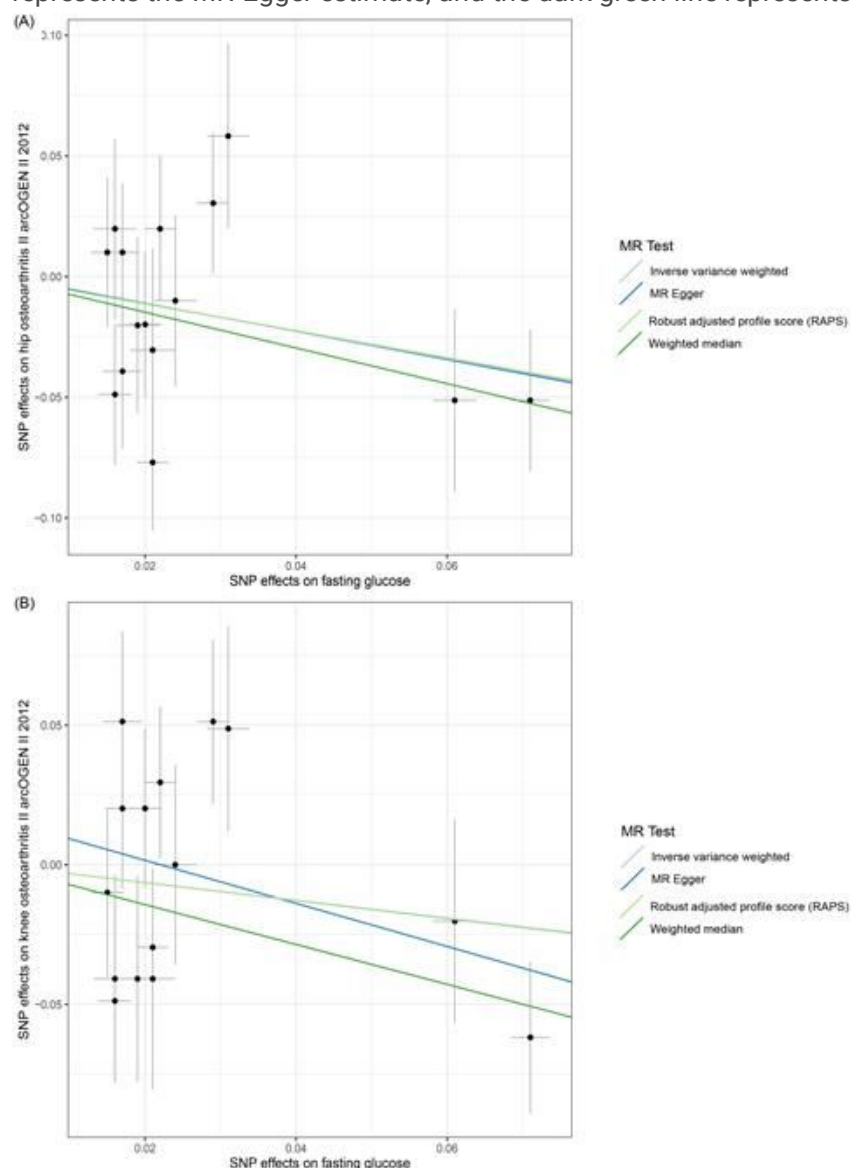

Figure 2

Scatter plots of genetic associations with fasting glucose against osteoarthritis using different MR methods. A) fasting glucose and hip osteoarthritis results; B) fasting glucose and knee osteoarthritis results. The slopes of each line represent the causal association for each method. The light blue line represents the inverse variance weighted estimate, the light green line represents the MR.RAPS estimate, the dark blue line represents the MR-Egger estimate, and the dark green line represents the weighted median estimate. 

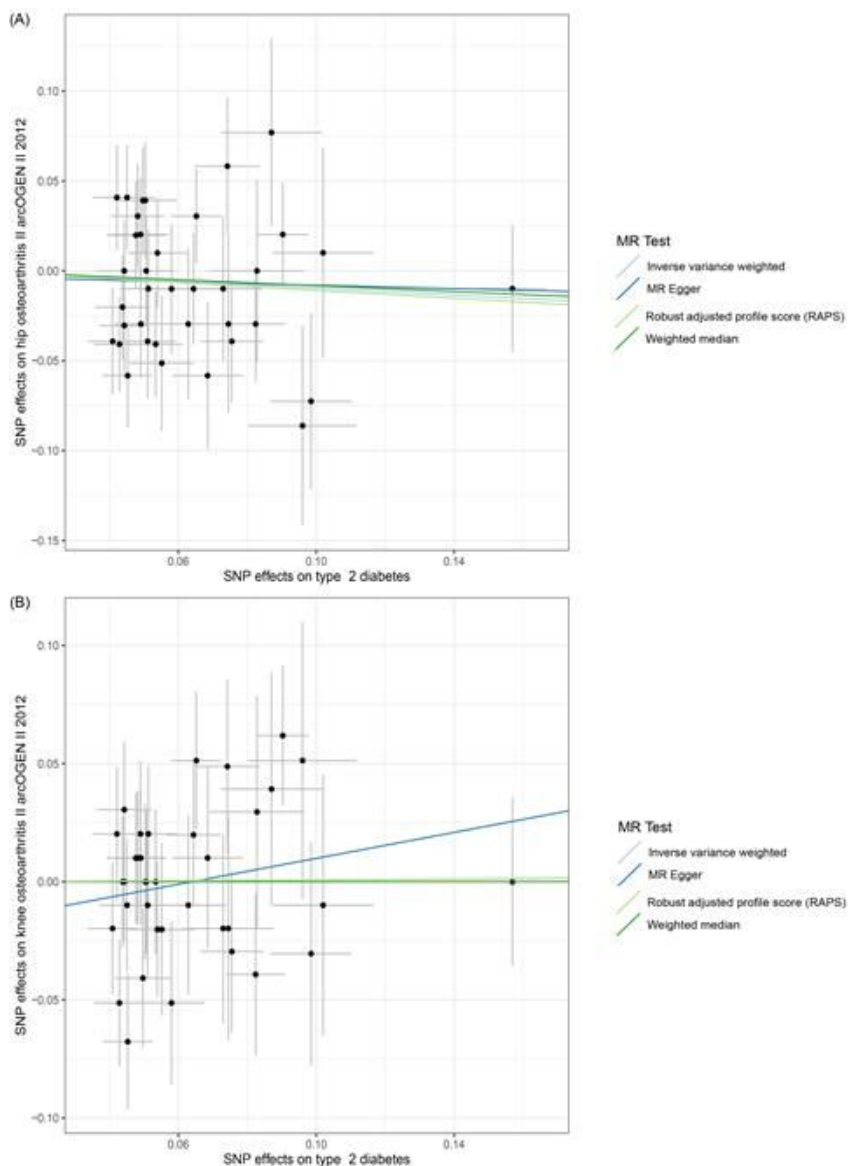

Figure 3

Scatter plots of genetic associations with type 2 diabetes risk against osteoarthritis using different MR methods. A) type 2 diabetes and hip osteoarthritis results; B) type 2 diabetes and knee osteoarthritis results. The slopes of each line represent the causal association for each method. The light blue line represents the inverse variance weighted estimate, the light green line represents the MR.RAPS estimate, the dark blue line represents the MR-Egger estimate, and the dark green line represents the weighted median estimate.

\section{Supplementary Files}

This is a list of supplementary files associated with this preprint. Click to download.

- supplementarymaterial.pdf 